\title{
Clinical Profile of Indoor Patients in the Department of Physical Medicine and Rehabilitation, Chittagong Medical College Hospital
}

\author{
Aminuddin A Khan ${ }^{1}$ \\ Md. Zahangir Alam Chowdhury ${ }^{2}$ \\ Mohammad Moin Uddin ${ }^{1 *}$ \\ Nusrat Sultana ${ }^{3}$ \\ Ahsanul Hoque Chowdhury'
}

'Department of Physical Medicine and Rehabilitation Chittagong Medical College

Chittagong, Bangladesh.

${ }^{2}$ Department of Physical Medicine and Rehabilitation Chattagram Maa-O-Shishu Hospital Medical College Chittagong, Bangladesh.

${ }^{3}$ Department of Dermatology Chittagong Medical College Chittagong, Bangladesh.

\begin{abstract}
Background: This observational study done on the indoor patients of department of Physical Medicine and Rehabilitation, Chittagong Medical College Hospital, admitted during the year 2011. The objective of this study was to see the most prevalent diseases admitted in our department. Methods: Participants (225) were all the patients admitted during the period of 2011. Patients admitted more than once were recorded once only. Patient's data were taken from hospital records. Results: Spondyloarthropathies (SpA) comprised $18.66 \%$ of the patients. Total patient of SpA was 42. Among them AS was 47.62\%. Prolapsed lumbar disk (PLID) patients constituted $12.44 \%$ (28) of hospital admission. Twenty one $(9.33 \%)$ patients suffered from other causes lumbago-sciatica. Admitted RA patient number were 12 (5.33\%) during this year. Total twelve patients with bone TB (5.33\%) were admitted during 2011. Nine TB patients ( $75 \%$ of bone TB) had TB in the hip. Total Stroke patients admitted in that year were $9(4 \%)$. Among them $8(88.89 \%)$ were ischemic. Conclusion: SpA, PLID and other lumbago-sciatica patients comprised the major bulk of the patients in inpatient department of PM\&R. Musculoskeletal diseases were the commonest cause of hospital admission for rehabilitation. Neurological causes were next common. Inflammatory musculoskeletal diseases also formed a good number of patients.
\end{abstract}

Key words: Patient profile; rehabilitation indoor; musculoskeletal; neurologic.

\section{INTRODUCTION}

Most of the patients admitted in the Physical Medicine department are related to musculoskeletal and neurological disease and disabilities. Musculoskeletal conditions are not regarded as major diseases by the authorities. But no other group of disorders leads to higher prevalence of disability than rheumatological diseases. No other group of disorders leads to a higher financial burden than the diseases rheumatological cases ${ }^{1}$.

World Health Organization concluded, that "although the diseases that kill attract much of the public's attention, musculoskeletal or rheumatic diseases are the major cause of morbidity throughout the world, having a substantial influence on health and quality of life, and inflicting an enormous burden of cost on health systems". In that publication, the following data are presented: (a) low back pain affects about $80 \%$ of people at some time of their life; (b) $40 \%$ of people over 70 years of age have osteoarthritis (OA) of the knee, with $25 \%$ of those with OA unable to perform daily activities of life and an additional $55 \%$ having some disability; (c) $1.1 \%$ of Norwegians $>60$ years have rheumatoid arthritis; and $(d)$ about two million people worldwide have hip fractures, usually associated with osteoporosis ${ }^{2}$.

The most recent statistics on Austrian health care show that in 2001 musculoskeletal conditions led to 8.4 million days of sick leave (more than 1 day per person living in the country, from newborns to the aged); in comparison, cardiovascular conditions accounted for 1.7 million days of sick leave, and even respiratory disorders, which include flu-like syndromes, did not top the rheumatic diseases ${ }^{3}$.

\section{Dr. Mohammad Moin Uddin} Department of Physical Medicine and Rehabilitation Chittagong Medical College Chittagong, Bangladesh. Mobile: +8801711159038 E-mail: moinmonju@gmail.com 
One specific epidemiological point needs to be made in relation to neurological disability. Neurological disease or injury accounts for the vast majority of patients with severe disability. Specialists with training in neurology and disability are obviously needed to help manage this large and important population of patients who have particular problems not seen in patients with non-neurological diseases ${ }^{4}$.

Neurological disorders have certain notable characteristics, e.g. they are large in number, and neurological disorders are a major cause of disability and account for a high proportion of severely disabled people under the age of 65 . More recent data related to costs in England for 1992-3 indicate that stroke was the second most expensive disorder treated in hospital ${ }^{5}$.

Objectives

To see the most common causes of hospital admission for pain management in the department of PM\&R, $\mathrm{CMCH}$.

Rationale

MSK diseases are very common causes of disability within the community but these diseases are neglected by everyone ${ }^{6-9}$. Hence, knowledge of the pattern of diseases is a vital need for proper management.

\section{Methodology}

\section{Study settings}

The study was carried out in the department of PM\&R, $\mathrm{CMCH}$. The inauguration of indoor in this department was done on $24^{\text {th }}$ Aigust, 2010. This was a descriptive type of study. Follow-up with no patients was done.

\section{Samples and data collection}

During the year 2011, total 326 patients were admitted to the inpatient department of PM\&R, CMCH. Among which, data were collected from hospital register about only 225 patients. Other patients could not be included due to incomplete data. So, all the patients admitted in inpatient department of $\mathrm{PM} \& \mathrm{R}, \mathrm{CMCH}$ in 2011 with complete data were included. However, patients admitted twice or more were included once only.

\section{Data handling}

After taking raw documents from hospital register, data were checked for completeness and correctness. Data were then tallied manually and frequencies were calculated. MS Excel was used for presentation of outcome picture.

\section{RESULTS}

Total 255 patients were taken into account. Among them, 157 $(61.56 \%)$ were males.

Table 1 delineates the spectrum of disease profile in the inpatient department in 2011 .
Table 1: Indications of hospital admission.

\begin{tabular}{llcccc} 
SL & Disease & \multicolumn{3}{c}{ Frequency } & Percent \\
& & Male & Female & Total & $(\%)$ \\
\hline 1 & Spondyloarthropathies & 38 & 4 & 42 & 18.66 \\
2 & Prolapsed Lumbar Disk & 20 & 8 & 28 & 12.44 \\
3 & Other lumbago-sciatica & 15 & 6 & 21 & 9.33 \\
4 & Mechanical Back Pain & 8 & 6 & 14 & 6.22 \\
5 & R.A & 1 & 11 & 12 & 5.33 \\
6 & Bone TB & 8 & 4 & 12 & 5.33 \\
7 & Osteoporosis & 5 & 6 & 11 & 4.89 \\
8 & OA Knee & 7 & 3 & 10 & 4.44 \\
9 & Stroke & 8 & 1 & 9 & 4 \\
10 & GBS, peripheral neuropathy & 8 & 1 & 9 & 4 \\
11 & Muscular Dystrophy & 5 & 1 & 6 & 2.67 \\
12 & Myelopathy & 6 & 0 & 6 & 2.67 \\
13 & Cervical spondylosis & 3 & 1 & 4 & 1.78 \\
14 & MND & 4 & 0 & 4 & 1.78 \\
15 & JIA & 2 & 1 & 3 & 1.33 \\
16 & Gout & 2 & 0 & 2 & 0.89 \\
17 & Others & 17 & 14 & 31 & 13.78 \\
\hline
\end{tabular}

Out of 42 SpA patients, $38(90 \%)$ were males. Of these, ankylosing spondylitis (AS) was 20 (47.62\%), reactive arthritis 4 , enteropathic 1 and undifferentiated $1335.71 \%$ ). Out of 49 lumbago-sciatica patients 28 had sciatica due to prolapsed lumbar disk. Out of 12 bone TB 8 were males, 9 (75\%) had TB in hip and $3(25 \%)$ had TB in the spine. Total Stroke patients admitted in last year were 9. Among them 8 (88.89\%) were ischemic and 8 patients were male $(88.89 \%)$ patients. Two thirds of the stroke patients had left hemiparesis and remainder had right hemiparesis. During one year period, 7 patients with muscle dystrophy were admitted (Figure 1).

Figure 1 : Pie chart presenting the frequencies of muscle dystrophies

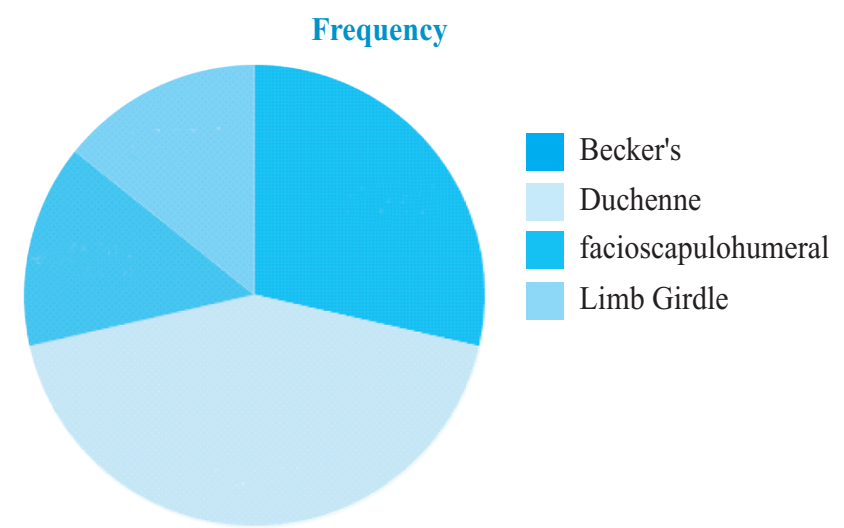

\section{DISCUSSION}

A of total 225 patients were analyzed from the admitted patients during this period. The majority were diagnosed as spondyloarthropathies (18.66\%). Second frequent patient's group was prolapsed lumber disk (12.44\%). But, PLID and other lumbago-sciatica together formed larger bulk of patients (around 22\%). LBP constituted the largest group (40\%) if SpA, sciatica, PLID and mechanical back pain are considered in this group. Why lumbago-sciatica is so common in our country is a matter of further study. 
Inflammatory joint disease was in 67 patients $(25 \%)$ which indicate that inflammatory musculoskeletal diseases are a big cause of disability. Neurologic causes of disability were not small. Total 34 patients got admitted with neurologic symptoms. $70.5 \%$ of all causes of disability and hospital admission consisted of musculoskeletal diseases. Maximum patients were males because we had more male beds than female beds. Here we took only 9 stroke patients. It is a very small number because most such patients receive the outdoor facilities of PM\&R department, $\mathrm{CMCH}$.

\section{CONCLUSION}

Musculoskeletal diseases were the commonest cause of hospital admission for rehabilitation in our department. Neurological causes were next common. Lumbago-sciatica formed the greatest bulk of patient then SpA constituted the second largest bulk. Inflammatory musculoskeletal diseases also formed a good number of patients.

\section{Limitations}

The present study was a small scale study done on a tertiary level hospital in the department of PM\&R, CMCH. Therefore, it did not reflect the actual incidence or prevalence of MSK problems. But we get a rough idea about the diseases that cause disability and hospital admission.

\section{Acknowledgements}

We show our gratitude to all our patients. We are grateful to all our teachers, colleagues and alliance health workers for their kind cooperation.

\section{DISCLOSURE}

All the authors declared no competing interest.

\section{REFERENCES}

1. Smolen JS. Combating the burden of musculoskeletal conditions. Ann Rheum Dis 2004; 63:329 doi:10.1136/ard.2004.022137.

2. WHO Scientific Group on the Burden of Musculoskeletal Conditions at the Start of the New Millennium. The burden of musculoskeletal conditions at the start of the new millennium: report of a WHO Scientific Group. Geneva: World Health Organisation, 2003.

3. Statistik Austria. Jahrbuch der Gesundheitsstatistik 2001. Vienna: Statistik Austria, 2003.

4. Wade DT. Epidemiology of disabling neurological disease: how and why does disability occur? J Neurol Neurosurg Psychiatry 1997; 63:11-18 doi:10.1136/jnnp.63.2008.11.

5. Richard Langton Hewer LR. The economic impact of neurological illness on the health and wealth of the nation and of individuals. J Neurol Neurosurg Psychiatry 1997; 63:19-23 doi:10.1136/jnnp.63.2008.19S.

6. Dacre JE, Griffith SM, Jolly BC. Rheumatology \& Medical Eduction in Great Britain, British Journal of Rheumatology 1996; 35: $269-274$

7. Dequeker J, Rasker H. High prevalence and impact of rheumatic diseases is not reflected in the medical curriculum: The ILAR Undergraduate Medical Education in Rheumatology (UMER) 2000 Project. Together everybody achieves more. J Rheumatol 1999; 25: 1037-40.

8. Smolen JS. Combating the burden of musculoskeletal diseases. Ann Rheum Dis 2004; 63: 329.

9. Freedman KB, Bernstein J. Educational deficiencies in musculoskeletal medicine. J Bone Joint Surg Am 2002; 84: 604-8. 